\title{
Incidence and clinical impact of respiratory viruses in adults with cystic fibrosis
}

\author{
William G Flight, ${ }^{1,2}$ Rowland J Bright-Thomas, ${ }^{1,2}$ Peter Tilston, ${ }^{3}$ Kenneth J Mutton, ${ }^{2,3}$ \\ Malcolm Guiver, ${ }^{3}$ Julie Morris, ${ }^{1}$ A Kevin Webb, ${ }^{1,2}$ Andrew M Jones ${ }^{1,2}$
}

\begin{abstract}
- Additional material is published online only. To view please visit the journal online (http://dx.doi.org/10.1136/ thoraxjnl-2013-204000).

${ }^{1}$ University Hospital of South Manchester NHS Foundation Trust, Manchester, UK ${ }^{2}$ Institute of Inflammation and Repair, University of Manchester, Manchester, UK ${ }^{3}$ Department of Virology, Central Manchester University Hospitals NHS Foundation Trust, Manchester, UK
\end{abstract}

\section{Correspondence to} Dr William G Flight, University Hospital of South Manchester NHS Foundation Trust, Southmoor Road, Manchester M23 9LT, UK; williamflight@ hotmail.com

Received 10 June 2013 Revised 9 September 2013 Accepted 20 September 2013 Published Online First 14 October 2013
To cite: Flight WG, BrightThomas RJ, Tilston $\mathrm{P}_{\text {, et al. }}$ Thorax 2014;69:247-253.
ABSTRACT
Background Viral respiratory infection (VRI) is a common cause of pulmonary exacerbations in children with cystic fibrosis (CF). The importance of VRI in adult CF populations is unclear.

Objective To determine the incidence and clinical impact of VRI among adults with CF.

Methods One hundred adults with CF were followed up prospectively for 12 months. Sputum, nose swabs and throat swabs were collected every 2 months and at onset of pulmonary exacerbation. PCR assays for adenovirus, influenza $A \& B$, human metapneumovirus, parainfluenza $1-3$, respiratory syncytial virus and human rhinovirus were performed on each sample. Symptom scores, spirometry and inflammatory markers were measured at each visit.

Results One or more respiratory viruses were detected in 191/626 (30.5\%) visits. Human rhinovirus accounted for $72.5 \%$ of viruses. Overall incidence of VRI was 1.66 (95\% Cl 1.39 to 1.92 ) cases/patient-year. VRI was associated with increased risk of pulmonary exacerbation $(\mathrm{OR}=2.19 ; 95 \% \mathrm{Cl} 1.56$ to $3.08 ; \mathrm{p}<0.001)$ and prescription of antibiotics (OR=2.26; $95 \% \mathrm{Cl} 1.63$ to 3.13; $p<0.001)$. Virus-positive visits were associated with higher respiratory symptom scores and greater C-reactive protein levels. Virus-positive exacerbations had a lower acute fall in $\mathrm{FEV}_{1}$ than virus-negative exacerbations ( $12.7 \%$ vs $15.6 \% ; p=0.040)$. The incidence of exacerbations, but not VRI, was associated with greater lung function decline over 12 months $(-1.79 \%$ per pulmonary exacerbation/year; $95 \% \mathrm{Cl}$ -3.4 to $-0.23 ; p=0.025$ ).

Conclusion VRI is common in adults with CF and is associated with substantial morbidity. Respiratory viruses are a potential therapeutic target in CF lung disease.

\section{INTRODUCTION}

Viral respiratory infections (VRIs) such as the common cold are associated with substantial morbidity among the general population. ${ }^{1}{ }^{2}$ Respiratory viruses are of even greater significance for people with chronic airways diseases and are implicated in exacerbations of both asthma and chronic obstructive pulmonary disease. ${ }^{3-8}$

Studies in children with cystic fibrosis (CF) suggest an incidence of up to five cases of VRI per patient-year. Viral infections in this population are associated with increased respiratory symptoms and disease progression. ${ }^{9-13} \mathrm{Up}$ to $64 \%$ of pulmonary exacerbations in paediatric CF populations are associated with respiratory viruses, ${ }^{11}$ yet the literature on VRI in adults with CF is sparse. Small studies in

\section{Key messages}

\section{What is the key question?}

- How common are viral respiratory infections (VRIs) among adults with cystic fibrosis (CF) and what clinical impact do such infections have?

\section{What is the bottom line?}

- Adults with CF have an average of 1-2 viral infections per year; these infections are strongly associated with pulmonary exacerbations, increased respiratory symptoms, raised inflammatory markers and a greater requirement for antibiotic therapy.

\section{Why read on?}

- This study describes detailed epidemiological, clinical and microbiological characteristics of VRIs in adults with CF. Respiratory viruses represent an underexploited therapeutic target in CF lung disease.

adults have identified a respiratory virus in up to $68 \%$ of pulmonary exacerbations, while case reports show that viruses such as influenza have the potential to cause severe respiratory infection in this patient group. ${ }^{14-17}$ There is also increasing in vitro evidence to demonstrate interactions between respiratory viruses, bacteria and host immune defences, which may have direct relevance to the pathogenesis of $\mathrm{CF}$ lung disease. ${ }^{18} 19$

To date, however, no large-scale, longitudinal study has been carried out to examine respiratory viruses in adult patients with CF. We performed a single-centre, prospective study to determine the incidence and clinical impact of VRI in a cohort of adults with CF. Preliminary data from this study have previously been presented in abstract form. ${ }^{20}$

\section{METHODS}

\section{Participants and study design}

Patients aged $\geq 18$ years attending the Manchester Adult Cystic Fibrosis Centre were invited to participate. The first 100 respondents were recruited. The only exclusion criterion was previous lung transplantation. Recruitment was completed between December 2010 and March 2011 and all patients gave written informed consent. The study was 
approved by the Greater Manchester West research ethics committee (reference 10/H1014/71).

Participants were followed up for 12 months or until death or lung transplantation. During follow-up, patients were seen routinely every 2 months. Between scheduled visits, patients were asked to contact the investigators when they developed symptoms of a pulmonary exacerbation (PEx) or upper respiratory tract infection (URTI). An additional study visit was then conducted within $48 \mathrm{~h}$. Clinicians were blinded to the results of virological tests when making treatment decisions at each study visit.

\section{Detection of respiratory viruses}

Sputum, nose swabs and throat swabs were collected at each visit for virological analysis. Sputum was expectorated spontaneously by the patient into sterile containers. Flocked swabs were collected from the nose and posterior pharynx and conveyed in viral transport medium at room temperature. Sputum or viral transport medium was mixed in a $1: 1$ ratio with AL lysis buffer (Qiagen, Hilden, Germany) before being inactivated at $80^{\circ} \mathrm{C}$ for $20 \mathrm{~min}$. Total nucleic acids were extracted using the QIAamp Virus Biorobot MDx instrument (Qiagen, Hilden, Germany), in accordance with the manufacturer's instructions. In-house PCR assays were performed to identify the presence of adenovirus, influenza $\mathrm{A} \& \mathrm{~B}$, human metapneumovirus, parainfluenza 1-3, respiratory syncytial virus and human rhinovirus. Details of the PCR assays, primers and probes are given in the online supplementary table S1. PCR cycle threshold (CT) values of $<45$ were considered positive. Lower CT values indicate that fewer cycles of PCR amplification were needed to detect viral nucleic acids and, therefore, reflect a stronger degree of PCR positivity.

\section{Genetic sequencing of rhinoviruses}

Genetic sequencing was performed on a subset of rhinoviruses identified during the study. All rhinovirus-positive sputum samples with a CT value $<40$ were selected for sequencing as well as upper airway swabs from patients with two or more consecutive rhinovirus-positive visits. Total nucleic acids were extracted as described above. Sequencing of the rhinovirus $5^{\prime}$ untranslated region was performed using the Applied Biosystems 3130xl Genetic Analyzer (Life Technologies Ltd, Paisley, UK) following a modification of the method reported by Lee et al, ${ }^{21}$ which is described in detail in the online supplementary appendix. Rhinovirus sequences were identified through phylogenetic analysis and comparison with the GenBank database using BLASTn. Sequences have been deposited with GenBank under accession numbers KF112083-KF112142.

\section{Clinical parameters}

PEx was defined according to a modification of the criteria described by Fuchs et $a l^{22}$ : four or more symptoms and a requirement for intravenous or oral antibiotics was considered an exacerbation. URTI symptoms were quantified using the score described by Johnston et al. ${ }^{3}$ Spirometry was performed at each visit using the Vitalograph 2150 volumetric spirometer (Vitalograph Ltd, Buckingham, UK) in line with accepted standards. ${ }^{23}$ Baseline forced expiratory volume in $1 \mathrm{~s}\left(\mathrm{FEV}_{1}\right)$ was taken as the best value in the 3 months before the study and compared with the best value in the 3 months after study completion. Full blood count and serum C-reactive protein levels were measured at each visit.

\section{Statistical analysis}

The sample size was determined using McNemar's test, assuming significance at the 5\% level. Ninety-four patients gave $80 \%$ power to detect a $20 \%$ difference in the proportion of viruspositive events among exacerbation visits compared with stable visits. The power calculation assumed that a third or more visits were positive for a virus overall. A recruitment target of 100 patients was set to allow for patient drop-out. The incidence of VRI is reported as the mean number of virus-positive visits per person-year and was calculated using a univariable Poisson regression model offset by the natural log of follow-up duration. Patients followed up for $<6$ months were excluded from incidence calculations. A multivariable Poisson regression model was used to evaluate the effect of demographic variables on the incidence of VRI. The variables included in this model were selected a priori based on factors known to increase the risk of VRI in the general population or to be of prognostic importance in CF.

Analysis of longitudinal outcomes used methods which take account of repeated observations from individual participants. Assessment of the association between VRI and other clinical outcomes used generalised estimating equation models employing an exchangeable correlation structure. Generalised estimating equation models with a logistic regression structure were selected for binary outcomes while a linear regression structure was used for continuous variables. Multiple linear regression was used to assess the impact of PEx and VRI on the rate of decline in $\mathrm{FEV}_{1}$. Univariable analyses were conducted in addition to a multivariable model adjusted for age and sex. Data are presented as mean (SD) or median (IQR), as appropriate. Variables which were not normally distributed were subject to log-transformation before inclusion in statistical models and this is highlighted in the manuscript where it has been used. A significance level of 0.05 was assumed unless stated otherwise. Statistical analysis was performed using SPSS V.20.0 (IBM, New York, USA).

\section{RESULTS}

\section{Patient demographics}

One hundred patients were recruited. Baseline demographics of the participants are detailed in table 1 . One participant was lost to follow-up and one withdrew from the study after the baseline visit. The remaining 98 patients completed a mean of 6.6 (SD 1.7) study visits over 11.5 (SD 2.0) months. Four patients were followed up for $<6$ months leaving 94 patients in the VRI incidence calculations. Three patients died during the study and

Table 1 Baseline demographics of study participants

\begin{tabular}{lc}
\hline Demographics & Value \\
\hline Number of patients & 100 \\
Age (years) & $28(23-36)$ \\
BMI $\left(\mathrm{kg} / \mathrm{m}^{2}\right) \dagger$ & $21.8(2.9)$ \\
Best FEV $\%$ in previous 12 months $\dagger$ & $59.3(22)$ \\
Female (\%) & 52 \\
F508del homozygous (\%) & 58 \\
Children <18 years at home (\%) & 7 \\
CF-related diabetes mellitus (\%) & 41 \\
Chronic Pseudomonas aeruginosa infection (\%) & 73 \\
Chronic Burkholderia cepacia complex infection (\%) & 14 \\
\hline *Median (IQR); tmean (SD). & \\
BMI, body mass index; CF, cystic fibrosis; FEV ${ }_{1}$, forced expiratory volume in $1 \mathrm{s.}$
\end{tabular}


Figure 1 Breakdown of viruses identified during the study. RSV, respiratory syncytial virus.

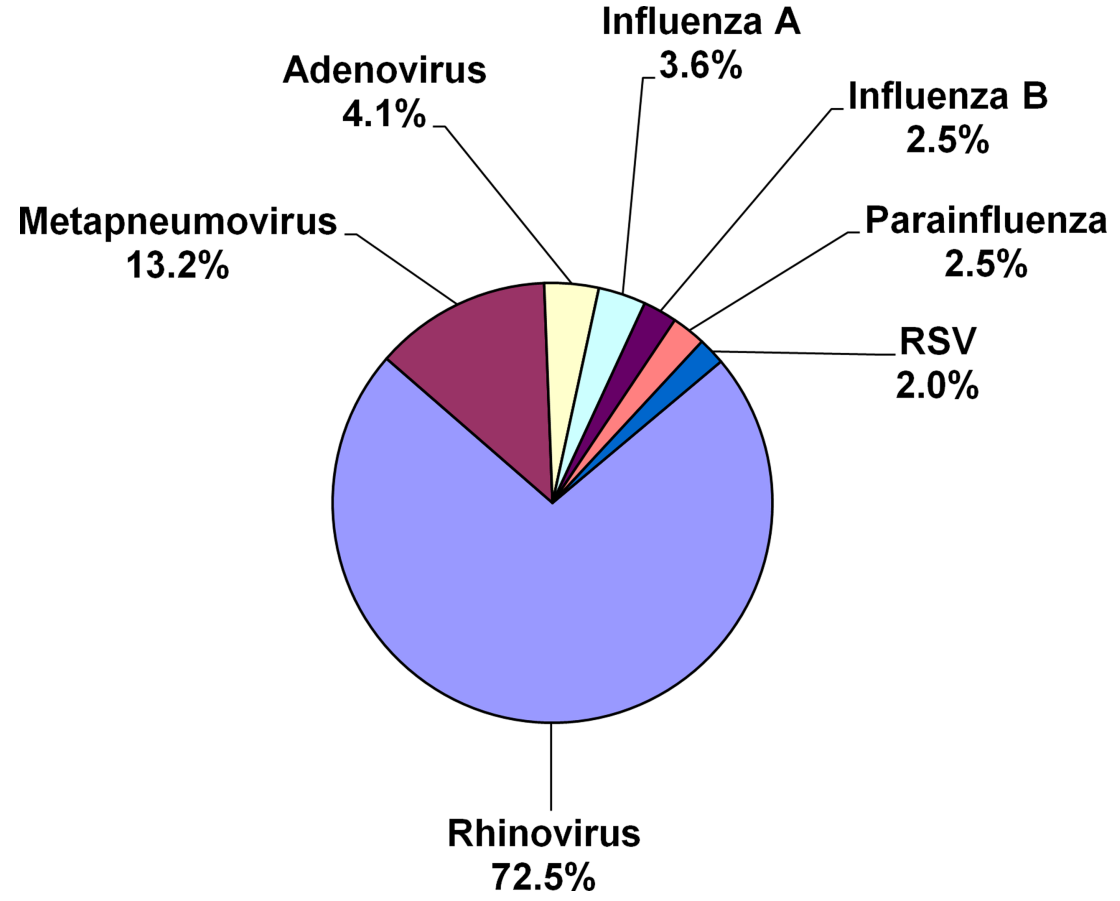

one underwent bilateral lung transplantation. Uptake of the seasonal influenza vaccination in the autumn before the study was $89 \%$.

\section{Identification of respiratory viruses}

Virology results were available for 626/649 completed study visits. Of these, 191 (30.5\%) were positive for a respiratory virus, including nine episodes of dual viral infection. Rhinovirus accounted for $72.5 \%$ of viruses identified. The distribution of viruses detected is shown in figure 1 . Details of the dual viral infections are given in online supplementary table S2.

The overall incidence of VRI was 1.66 (95\% CI 1.39 to 1.92$)$ cases/patient-year. Rhinovirus had the highest incidence at 1.17 (95\% CI 0.95 to 1.39 ) cases/patient-year. Equivalent values for other viruses were: metapneumovirus 0.28 (0.17 to 0.39$)$, influenza A 0.08 (0.02 to 0.13), adenovirus 0.08 (0.02 to 0.13 ), influenza B 0.05 (0.01 to 0.10$)$, parainfluenza virus $1-30.05$ (0.01 to 0.10$)$ and respiratory syncytial virus 0.04 (0.001 to 0.09 ) cases/patient-year. Eighty of 94 patients (85\%) had at least one episode of laboratory-confirmed VRI during the study. Demographic variables did not have a significant effect on the incidence of VRI (see table 2).

Table 2 Demographic risk factors for viral respiratory infection

\begin{tabular}{llll}
\hline Risk factors & Rate ratio* & $\mathbf{9 5 \%} \mathbf{C l}$ & $\mathbf{p ~ V a l u e ~}$ \\
\hline Female gender & 1.07 & 0.73 to 1.57 & 0.731 \\
Age & 1.00 & 0.98 to 1.02 & 0.689 \\
F508del homozygous & 1.29 & 0.88 to 1.84 & 0.199 \\
Baseline FEV ${ }_{1}$ & 1.01 & 0.80 to 1.29 & 0.907 \\
Chronic Pseudomonas aeruginosa & 0.90 & 0.61 to 1.32 & 0.590 \\
CF-related diabetes & 1.15 & 0.80 to 1.65 & 0.468 \\
Children <18 years at home & 1.14 & 0.71 to 1.81 & 0.591 \\
\hline
\end{tabular}

${ }^{*}$ Rate ratio calculated using a Poisson regression model.

$\mathrm{CF}$, cystic fibrosis; $\mathrm{FEV}_{1}$, forced expiratory volume in $1 \mathrm{~s}$.

\section{Genetic sequencing of rhinovirus strains}

The rhinovirus $5^{\prime}$ untranslated region was sequenced successfully from 61 respiratory tract specimens known to be PCR positive for rhinovirus. These samples were collected at 42 study visits from 27 separate patients and comprised 29 sputum specimens, 21 nose swabs and 11 throat swabs. Thirty-five different rhinovirus subtypes were identified in total. Human rhinovirus A was detected at 29/42 visits (69.0\%) with 11/42 visits $(26.2 \%)$ positive for human rhinovirus B. Human rhinovirus $\mathrm{C}$ was detected in only $2 / 42(4.8 \%)$ visits. There were no cases of simultaneous infection with different rhinovirus subtypes. One patient appeared to harbour the same strain of rhinovirus A at three separate study visits over a period of 10 months. No other cases of persistent rhinovirus infection were identified in the cohort. Figure 2 shows a phylogenetic tree to illustrate the diversity of rhinoviruses sequenced in this study.

Rhinovirus A infection seemed to have more severe clinical outcomes than rhinovirus B (see online supplementary table S3). Rhinovirus A-positive visits had greater URTI scores (12.5 (SD 7.1) vs 6.5 (SD 5.6); $\mathrm{p}=0.014)$ and higher $\mathrm{C}$-reactive protein levels (geometric mean 15.9 (range 0-140) vs 6.0 (2-24); $\mathrm{p}=0.008$ ) than rhinovirus B-positive episodes.

\section{Association between VRI and pulmonary exacerbation (PEx)}

Data were available for both exacerbation and virology status in 609 visits. Of these, $229(37.6 \%)$ episodes met the predefined criteria for PEx. Patients had a mean of 2.6 (SD 1.8) exacerbations/patient-year. Forty per cent of exacerbations were positive for a virus compared with $24 \%$ of non-exacerbation visits. Identification of a virus was associated with increased risk of PEx $(\mathrm{OR}=2.19 ; 95 \%$ CI 1.56 to $3.08 ; \mathrm{p}<0.001)$. Overall, 306/ $649(47 \%)$ visits resulted in a prescription for antibiotics, of which $49 \%$ were for intravenous antibiotics. Virus-positive episodes were associated with a greater likelihood of any antibiotic prescription $(\mathrm{OR}=2.26 ; 95 \%$ CI 1.63 to 3.13 ; $\mathrm{p}<0.001)$ but there was no significant increase in the prescription of intravenous antibiotics $(\mathrm{OR}=1.30 ; 95 \% \mathrm{CI} 0.94$ to $1.81 ; \mathrm{p}=0.114)$. 


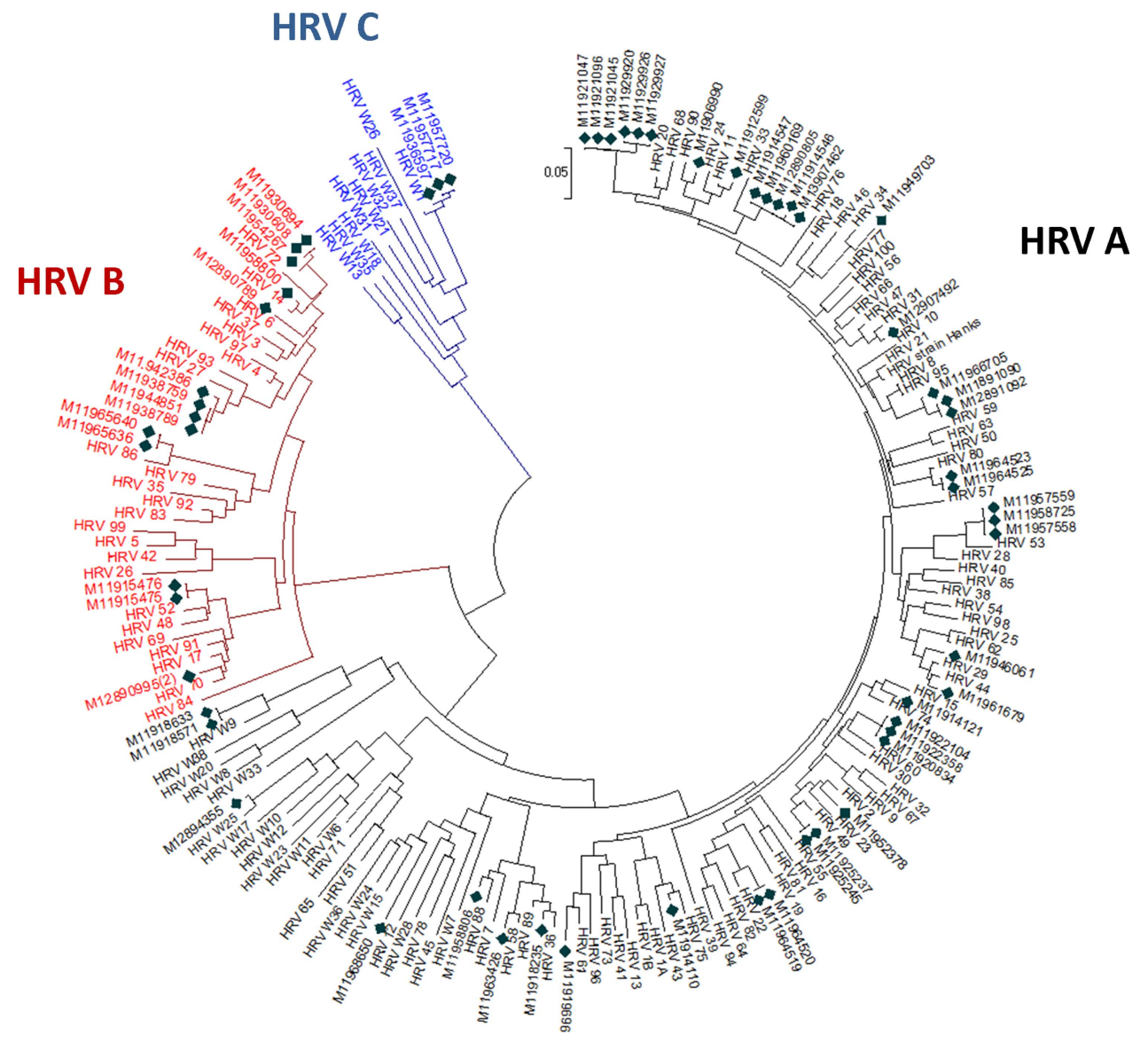

Figure 2 Phylogenetic tree showing rhinoviruses detected in clinical samples from this study and reference rhinovirus strains reported by Lee et al. ${ }^{22}$ The phylogenetic tree was constructed in MEGA 5.1 using the neighbour joining method with bootstrap values of 500 . $\$$ indicates study samples. HRV, human rhinovirus.

The mean PCR CT value for virus-positive specimens was 37.1 (SD 5.8) cycles, with lower CT values indicating a stronger degree of PCR positivity. Lower CT values were a significant predictor of meeting the criteria for PEx $(\mathrm{OR}=0.92 ; 95 \% \mathrm{CI}$ 0.88 to $0.97 ; \mathrm{p}<0.001)$ and for prescription of antibiotics $(\mathrm{OR}=0.89 ; 95 \%$ CI 0.83 to $0.95 ; \mathrm{p}<0.001)$.

\section{Impact of VRI on acute and long-term changes in FEV}

The mean difference between the $\mathrm{FEV}_{1}$ at baseline and a given study visit was similar at virus-positive and virus-negative episodes (8.7 vs 9.4\%; $p=0.412)$. When exacerbations were considered separately, virus-positive PEx were associated with a smaller fall in $\mathrm{FEV}_{1}$ than virus-negative PEx (12.7\% (SD 1.3) vs 15.6\% (SD $1.4) ; \mathrm{p}=0.040)$.
In comparison with baseline values, the end of study $\mathrm{FEV}_{1}$ fell by a mean of $1.5 \%$ (SD 12.4). There was no evidence of a significant association between the number of virus-positive visits per year and an increased rate of decline in $\mathrm{FEV}_{1}$ over the study period $(-0.88 \%$ per VRI/year; $95 \%$ CI -2.7 to +0.95 ; $\mathrm{p}=0.268$ ). The number of PEx per year did have a significant association with decline in $\mathrm{FEV}_{1}$, which persisted after adjustment for baseline demographics and number of viral infections (see table 3).

\section{Effect of VRI on symptoms and inflammatory markers}

Virus-positive visits were associated with a mean URTI score of 7.6 (SD 6.3) out of 27 compared with 5.3 (SD 4.6) for virusnegative visits $(p<0.001)$. Mean Fuchs PEx scores, from a

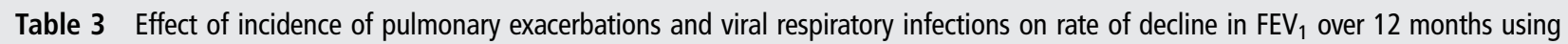
a multiple linear regression model

\begin{tabular}{|c|c|c|c|c|c|c|}
\hline & \multicolumn{3}{|c|}{ Univariable analysis } & \multicolumn{3}{|c|}{ Multivariable analysis } \\
\hline & $\Delta \mathrm{FEV}_{1}(\%)$ & $95 \% \mathrm{Cl}$ & p Value & $\Delta \mathrm{FEV}_{1}(\%)$ & $95 \% \mathrm{Cl}$ & p Value \\
\hline PEx per year & -1.65 & -3.1 to -0.23 & 0.024 & -1.79 & -3.4 to -0.23 & 0.025 \\
\hline Virus-positive visits per year & -0.88 & -2.7 to +0.95 & 0.268 & -0.49 & -2.3 to +1.4 & 0.600 \\
\hline Age & - & - & - & -0.024 & -0.3 to +0.3 & 0.876 \\
\hline Sex* & - & - & - & +3.4 & -1.9 to +8.7 & 0.210 \\
\hline
\end{tabular}


maximum value of 12, were 3.6 (SD 2.3) and 2.9 (SD 2.4) in the virus-positive and negative groups, respectively $(\mathrm{p}<0.001)$. The odds of virus-positive visits being associated with individual symptoms are shown in supplementary table S4. Increased cough, change in sputum, increased dyspnoea, sinus discharge, sore throat and hoarse voice were all significantly more likely at virus-positive visits. Among virus-positive visits, 22.8\% were minimally symptomatic as defined by a total of $<4$ in both the URTI and PEx scores. VRI was associated with higher C-reactive protein levels (geometric mean 10.3 (range 0-244) vs 8.4 (0-165); $\mathrm{p}=0.004)$ and lower lymphocyte counts $\left(1.83\right.$ vs $1.94 \times 10^{9}$; $\mathrm{p}=0.011)$ than virus-negative visits.

In comparison with visits positive for other viruses, rhinovirus infection was associated with a similar rate of PEx $(47.8 \%$ vs $48.6 \%$, respectively). Non-rhinovirus VRI was associated with an increased rate of prescription of intravenous antibiotics $(41.3 \%$ vs $21.0 \%$; $=0.011)$ but rhinovirus infection was otherwise comparable in severity to that of other viruses (see table 4).

\section{Diagnosis of VRI}

Complete sets of virology samples (ie, sputum, nose swabs and throat swabs) were collected at 469 visits. Samples were missing most commonly because the patient was unable to expectorate sputum. One or more viruses were detected at 151/469 (32.2\%) visits. Of these, $100(66.2 \%)$ were positive in only one of the three specimen types, $30(19.9 \%)$ in two and 21 (13.9\%) in all three specimen types. Sputum samples identified $58.0 \%$ of viruses compared with $46.7 \%$ for nose swabs and $43.3 \%$ for throat swabs. The sensitivity of different specimen combinations is shown in figure 3. Sputum samples identified a wider diversity of viral pathogens than upper airway swabs (see online supplementary figure S1).

Receiver operating characteristic analysis suggests that the URTI score has limited usefulness for the prediction of laboratory-confirmed VRI in adults with CF (see online supplementary figure S2). The original diagnostic threshold of $\geq 4 / 27$ gave a sensitivity of 0.68 (95\% CI 0.45 to 0.86$)$ and specificity of 0.51 ( 0.40 to 0.63$)$. Positive and negative predictive values were 0.28 and 0.85 , respectively, with an area under the receiver operating characteristic curve of 0.68 (95\% CI 0.54 to 0.81 ).

\section{DISCUSSION}

This paper presents the results of the first large-scale, prospective study to document the incidence of VRI in adults with CF. Our experience shows that respiratory viruses are common in this population and are strongly associated with pulmonary exacerbations and increased morbidity. The overall incidence of VRI in our study is consistent with previous paediatric CF studies and it is now clear that respiratory viruses have an important role in CF lung disease in adults as well as children. ${ }^{9-13} 24$

We identified human rhinovirus as overwhelmingly the most common viral pathogen in adults with CF. Rhinovirus belongs to the Picornaviridae family of viruses and is the principal cause of the common cold. ${ }^{25}$ For patients with airway diseases, rhinovirus is a greater problem and is associated with most acute asthma attacks. $^{3}$ The predominance of rhinovirus in our study mirrors data from children with CF and confirms that rhinovirus is an important CF pathogen. ${ }^{10-13}$ Our data also show that rhinovirus $\mathrm{A}$ is the predominant rhinovirus species to affect adults with CF. We have not detected a prominent role for the recently identified rhinovirus $\mathrm{C}$ species, which contrasts sharply with the findings of de Almeida and colleagues in their paediatric cohort. ${ }^{11}$ Our study has shown in $\mathrm{CF}$ for the first time that rhinovirus $\mathrm{A}$ has more severe clinical outcomes than rhinovirus $\mathrm{B}$, which may have implications for the development of future antirhinoviral treatments.

The relationship between pulmonary exacerbations, respiratory viruses and decline in lung function warrants close analysis. Most paediatric CF studies have found accelerated lung function decline in patients with more frequent viral infections. ${ }^{12} 1326$ We found that respiratory viruses double the risk of PEx and, separately, that the incidence of PEx is linked to an increased rate of $\mathrm{FEV}_{1}$ deterioration. Despite this, our data do not show a statistically significant link between incidence of VRI and lung function decline.

Explanations might include the fact that our study was not powered to detect changes in lung function and a larger study might have unveiled an effect of viral infections on $\mathrm{FEV}_{1}$ decline. In addition, respiratory viruses were associated with a wide spectrum of clinical severity in our study. The "noise" from asymptomatic viral infections might have masked the impact of more harmful viruses. Finally, the unusually low incidence of influenza over the winter of 2011-2012 and our high vaccine coverage rate might have had an impact on our results ${ }^{27}$ only one case of influenza was seen over the entire second winter of the study.

An important finding of this study is the detection at $24 \%$ of study visits of a respiratory virus which did not meet the prespecified criteria for PEx. The prevalence of VRI during clinical stability has not been reported before among adults with CF but the rate in paediatric patients has varied from 13 to $53 \%$. $^{10} 112428$

Table 4 Clinical outcomes for rhinovirus-positive visits in comparison with visits positive for other viruses

\begin{tabular}{|c|c|c|c|c|c|}
\hline Binary outcomes & Rhinovirus & Other viruses combined & $\mathrm{OR}$ & $95 \% \mathrm{Cl}$ & $p$ Value \\
\hline PEx (\%) & 48.6 & 47.8 & 1.10 & 0.60 to 2.06 & 0.740 \\
\hline Prescription of any $A B x(\%)$ & 59.4 & 65.2 & 0.92 & 0.46 to 1.83 & 0.808 \\
\hline Prescription of IV ABx (\%) & 21.0 & 41.3 & 0.38 & 0.18 to 0.80 & 0.011 \\
\hline Continuous outcomes* & Rhinovirus & Other viruses combined & Adjusted mean difference & $95 \% \mathrm{Cl}$ & $p$ Value \\
\hline URTI score & $7.9(6.6)$ & $6.9(5.6)$ & +0.96 & -0.96 to +2.89 & 0.328 \\
\hline PEx score & $3.6(2.4)$ & $3.6(2.4)$ & +0.06 & -0.69 to +0.80 & 0.884 \\
\hline Acute fall in $\mathrm{FEV}_{1} \%$ & $13.5(10.7)$ & $16.9(11.8)$ & -2.09 & -5.5 to +1.4 & 0.234 \\
\hline $\log \operatorname{CRP}(\mathrm{mg} / \mathrm{l}) \dagger$ & $2.20(1.4)$ & $2.21(1.3)$ & +0.03 & -0.32 to +0.38 & 0.866 \\
\hline White cell count $\left(\times 10^{9}\right)$ & $10.6(3.9)$ & $10.3(3.1)$ & -0.19 & -0.99 to +0.61 & 0.637 \\
\hline
\end{tabular}

Analysis used generalised estimating equation models with logistic regression for binary outcomes and linear regression for continuous variables. Non-rhinovirus viral respiratory infection was the comparator variable in each analysis.

*Continuous variables are reported as mean (SD).

tCRP values were log-transformed to correct for non-normal distribution.

$\mathrm{ABx}$, antibiotics; CRP, C-reactive protein; FEV 1 , forced expiratory volume in $1 \mathrm{~s}$; PEx, pulmonary exacerbation; URTI, upper respiratory tract infection. 
Figure 3 Sensitivity of different combinations of sputum, nose swabs and throat swabs for the diagnosis of viral respiratory infection in adults with cystic fibrosis.

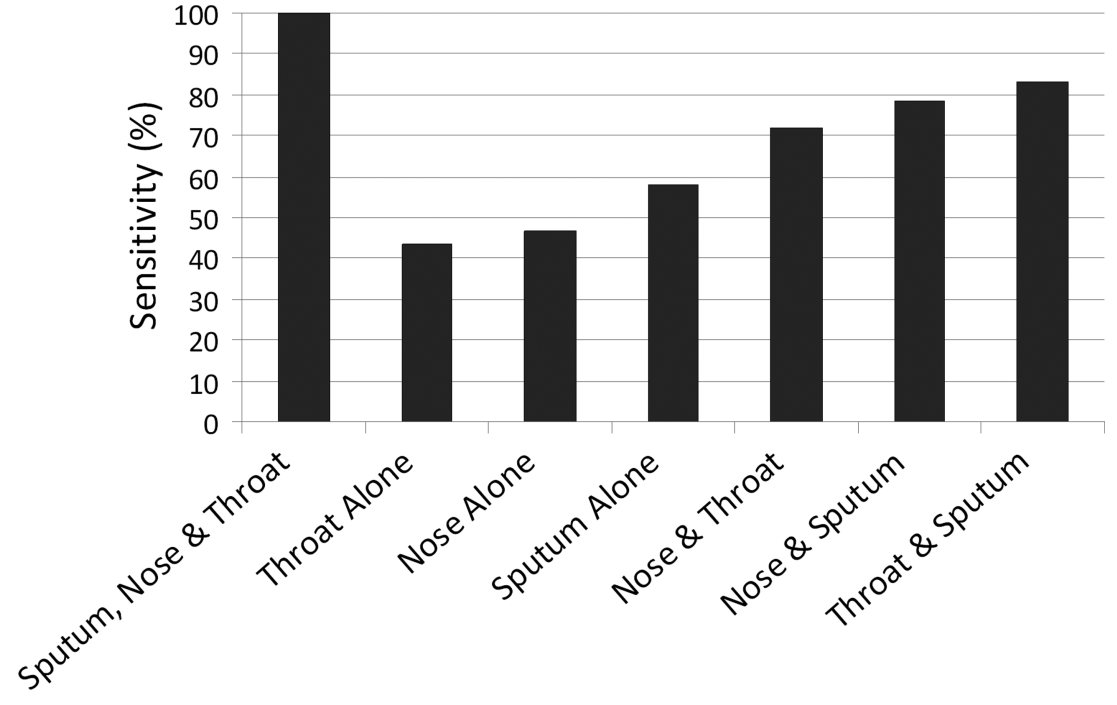

Our data are therefore broadly consistent with the paediatric CF literature. It is important to note that PEx is not a binary phenomenon. Acute deteriorations in CF lung disease represent a spectrum of events and the visits not meeting the criteria for PEx are likely to have included 'mild' exacerbations and pure upper respiratory viral infections.

Before this study, little was known about the symptom burden associated with respiratory viruses in adults with $\mathrm{CF}$. We have shown that VRI is associated with characteristic symptoms, including sore throat, sinus discharge and hoarse voice. Other symptoms associated with VRI included increased cough, change in sputum and dyspnea, which are extremely common in patients with CF. In general, many of the symptoms associated with VRI are non-specific and this probably explains the poor diagnostic accuracy of the URTI symptom score in our population. It should be noted that the Johnston URTI score was originally used in a paediatric asthma study and has not been validated for use in $\mathrm{CF}^{3}$ The score did have a high negative predictive value but our findings suggest that URTI symptoms should not be used as a surrogate for laboratory-confirmed VRI in clinical practice or future research in CF. Olesen et $a l^{24}$ reached similar conclusions among paediatric patients with $\mathrm{CF}$.

Our study has also presented new data on the optimal means of diagnosing VRI in CF. We have previously shown that CF sputum is suitable for the diagnosis of VRI using PCR. ${ }^{15}$ In contrast to a recent paediatric study, ${ }^{28}$ we found that sputum had higher viral identification rates than upper airway swabs and detected a greater variety of viruses. This presumably reflects the greater ease with which most adult patients expectorate sputum. Our study suggests that a combination of sputum and upper airway samples is required to maximise diagnostic sensitivity in adults with CF.

A number of limitations of this study need to be recognised and it is likely that our results underestimate the true incidence of VRI. First, our PCR panel of nine respiratory viruses is not exhaustive and did not include bocavirus, coronavirus or parainfluenza 4. In addition, the study design required patients to contact the CF centre at onset of new respiratory symptoms so mild illnesses might have gone unreported. The planned strategy of testing sputum, nose swabs and throat swabs was achieved in only $72 \%$ of study episodes. This reflects 'real-life' practice where patients often vary in their ability to produce sputum. Finally, we used the aggregate number of symptoms making up the Fuchs criteria as both a diagnostic tool and a surrogate marker for the severity of lower respiratory tract symptoms. The Fuchs criteria were not designed for use in this way but we took the pragmatic approach of assuming a correlation between the number of PEx symptoms and exacerbation severity.

Allowing for these limitations, our study has shown a clear link between respiratory viruses and exacerbations in adults with CF. PEx are extremely important events in CF and are linked to impaired quality of life, ${ }^{29}$ declining lung function ${ }^{30}$ and increased mortality. ${ }^{31} \mathrm{PEx}$ remain common despite the availability of established prophylactic treatments such as maintenance antibiotics and mucolytic agents. The need for additional treatments to prevent such exacerbations, therefore, is clear. Our findings suggest that respiratory viruses, especially rhinovirus, represent a potential therapeutic target in CF lung disease.

In summary, we have demonstrated that respiratory viruses are common pathogens in adults with CF and are strongly linked to pulmonary exacerbations. Further research needs to be directed towards understanding the pathophysiology of these infections and virus-bacteria interactions within the CF lung. Such studies may ultimately lead to effective new treatments to prevent virus-induced exacerbations in patients with CF.

Acknowledgements We are very grateful to Alan Lord, Chris Parker, Helen Flight and the MACFC nursing staff for assistance with the study.

Contributors WGF was involved in study design, data collection, data analysis and wrote the first draft. All other authors contributed to study design, data collection or analysis and all authors helped to revise the manuscript.

Funding The study was funded by the Manchester Adult Cystic Fibrosis Centre's charitable fund.

Competing interests None.

Ethics approval Greater Manchester West NHS research ethics committee.

Provenance and peer review Not commissioned; externally peer reviewed.

\section{REFERENCES}

1 Monto A, Ullman B. Acute respiratory illness in an American community. The Tecumseh study. JAMA 1974;227:164-9.

2 Fox J, Hall C, Cooney M, et al. The Seattle virus watch. II. Objectives, study population and its observation, data processing and summary of illnesses. Am J Epidemiol 1972;96:270-85.

3 Johnston SL, Pattemore PK, Sanderson G, et al. Community study of role of viral infections in exacerbations of asthma in 9-11 year old children. BMJ 1995;310:1225-9.

4 Freymuth F, Vabret A, Brouard J, et al. Detection of viral, Chlamydia pneumoniae and Mycoplasma pneumoniae infections in exacerbations of asthma in children. J Clin Virol 1999;13:131-9. 
5 Rakes GP, Arruda E, Ingram JM, et al. Rhinovirus and respiratory syncytial virus in wheezing children requiring emergency care. IgE and eosinophil analyses. Am J Respir Crit Care Med 1999;159:785-90.

6 Corne JM, Marshall C, Smith S, et al. Frequency, severity, and duration of rhinovirus infections in asthmatic and non-asthmatic individuals: a longitudinal cohort study. Lancet 2002;359:831-4.

7 Seemungal T, Harper-Owen R, Bhowmik A, et al. Respiratory viruses, symptoms, and inflammatory markers in acute exacerbations and stable chronic obstructive pulmonary disease. Am J Respir Crit Care Med 2001;164:1618-23.

8 Rohde G, Wiethege A, Borg I, et al. Respiratory viruses in exacerbations of chronic obstructive pulmonary disease requiring hospitalisation: a case-control study. Thorax 2003; 58:37-42.

9 van Ewijk B, van der Zalm M, Wolfs T, et al. Prevalence and impact of respiratory viral infections in young children with cystic fibrosis: prospective cohort study. Pediatrics 2008; 122:1171-6.

10 Wat $D$, Gelder $C$, Hibbitts $S$, et al. The role of respiratory viruses in cystic fibrosis. J Cyst Fibros 2008;7:320-8.

11 de Almeida MB, Zerbinati RM, Tateno AF, et al. Rhinovirus $C$ and respiratory exacerbations in children with cystic fibrosis. Emerg Infect Dis 2010;16:996-9.

12 Collinson J, Nicholson K, Cancio E, et al. Effects of upper respiratory tract infections in patients with cystic fibrosis. Thorax 1996;51:1115-22.

13 Smyth A, Smyth R, Tong C, et al. Effect of respiratory virus infections including rhinovirus on clinical status in cystic fibrosis. Arch Dis Child 1995;73:117-20.

14 Conway SP, Simmonds EJ, Littlewood JM. Acute severe deterioration in cystic fibrosis associated with influenza A virus infection. Thorax 1992;47:112-4.

15 Jones AM, Flight W, Isalska B, et al. Diagnosis of respiratory viral infections in cystic fibrosis by PCR using sputum samples. Eur Respir J 2011;38:1486-7.

16 Whitaker $P$, Etherington $C$, Denton $M$, et al. A/H1N1 flu pandemic. A/H1N1 and other viruses affecting cystic fibrosis. BMJ 2009;339.

17 Wark PA, Tooze M, Cheese $L$, et al. Viral infections trigger exacerbations of cystic fibrosis in adults and children. Eur Respir J 2012;40:510-2.

18 Chattoraj SS, Ganesan S, Jones AM, et al. Rhinovirus infection liberates planktonic bacteria from biofilm and increases chemokine responses in cystic fibrosis airway epithelial cells. Thorax 2011;66:333-9.
19 Van Ewijk B, Wolfs T, Aerts $\mathrm{P}$, et al. RSV mediates Pseudomonas aeruginosa binding to cystic fibrosis and normal epithelial cells. Pediatr Res 2007;61 398-403.

20 Flight WG, Mutton $\mathrm{K}$, Webb AK, et al. The incidence of respiratory virus infection in adults with cystic fibrosis. Pediatr Pulmonol 2012;47(S35):315.

21 Lee WM, Kiesner C, Pappas T, et al. A diverse group of previously unrecognized human rhinoviruses are common causes of respiratory illnesses in infants. PLOS ONE 2007;2:e966.

22 Fuchs $\mathrm{H}$, Borowitz D, Christiansen D, et al. Effect of aerosolized recombinant human DNase on exacerbations of respiratory symptoms and on pulmonary function in patients with cystic fibrosis. The Pulmozyme Study Group. N Engl J Med 1994;331:637-42.

23 Miller MR, Hankinson J, Brusasco V, et al. Standardisation of spirometry. Eur Respir J 2005;26:319-38.

24 Olesen H, Nielsen L, Schiotz P. Viral and atypical bacterial infections in the outpatient pediatric cystic fibrosis clinic. Pediatr Pulmonol 2006;41:1197-204.

25 Andrewes C. Rhinoviruses and common colds. Annu Rev Med 1966;17:361-70.

26 Wang E, Prober C, Manson B, et al. Association of respiratory viral infections with pulmonary deterioration in patients with cystic fibrosis. $N$ Engl J Med 1984;311:1653-8.

27 Surveillance of influenza and other respiratory viruses in the UK: 2011-2012 report. Health Protection Agency, 2012. http://www.hpa.org.uk/Publications/ InfectiousDiseases/Influenza/1206influannualreport/

28 Burns JL, Emerson J, Kuypers J, et al. Respiratory viruses in children with cystic fibrosis: viral detection and clinical findings. Influenza Other Respi Viruses 2012;6:218-23.

29 Britto $M$, Kotagal U, Hornung $R$, et al. Impact of recent pulmonary exacerbations on quality of life in patients with cystic fibrosis. Chest 2002;121:64-72.

30 Sanders DB, Bittner RC, Rosenfeld $\mathrm{M}$, et al. Failure to recover to baseline pulmonary function after cystic fibrosis pulmonary exacerbation. Am J Resp Crit Care Med 2010;182:627-32.

31 de Boer K, Vandemheen $\mathrm{KL}$, Tullis $\mathrm{E}$, et al. Exacerbation frequency and clinical outcomes in adult patients with cystic fibrosis. Thorax 2011;66:680-5. 